\title{
Wireless capsule endoscopy: a comparison with push enteroscopy in patients with gastroscopy and colonoscopy negative gastrointestinal bleeding
}

\author{
M Mylonaki, A Fritscher-Ravens, P Swain
}

\begin{abstract}
Background: The development of wireless capsule endoscopy allows painless imaging of the small intestine. Its clinical use is not yet defined. The aim of this study was to compare the clinical efficacy and technical performance of capsule endoscopy and push enteroscopy in a series of 50 patients with colonoscopy and gastroscopy negative gastrointestinal bleeding.

Methods: A wireless capsule endoscope was used containing a CMOS colour video imager, transmitter, and batteries. Approximately 50000 transmitted images are received by eight abdominal aerials and stored on a portable solid state recorder, which is carried on a belt. Push enteroscopy was performed using a $240 \mathrm{~cm}$ Olympus video enteroscope. Results: Studies in 14 healthy volunteers gave information on normal anatomical appearances and preparation. In 50 patients with gastrointestinal bleeding and negative colonoscopy and gastroscopy, push enteroscopy was compared with capsule endoscopy. A bleeding source was discovered in the small intestine in 34 of 50 patients $(68 \%)$. These included angiodysplasia (16), focal fresh bleeding (eight), apthous ulceration suggestive of Crohn's disease (three), tumour (two), Meckel's diverticulum (two), ileal ulcer (one), jejunitis (one), and ulcer due to intussusception (one). One additional intestinal diagnosis was made by enteroscopy. The yield of push enteroscopy in evaluating obscure bleeding was $32 \%(16 / 50)$. The capsule identified significantly more small intestinal bleeding sources than push Conclusions: In this study capsule endoscopy was superior to push enteroscopy in the diagnosis of recurrent bleeding in patients who had a negative gastroscopy and colonoscopy. It was safe and well tolerated.
\end{abstract}

See end of article for authors' affiliations

Correspondence to: Professor C P Swain Endoscopy Unit, Royal London Hospital, Whitechapel, London El 1BB, UK;

PaulSwain $1 @$ compuserve.com enteroscopy $(p<0.05)$. Patients preferred capsule endoscopy to push enteroscopy (p<0.001).

Accepted for publication 7 February 2003

$\mathrm{T}$ he invention of fibreoptic endoscopy ${ }^{1}$ has allowed inspection of the oesophagus, stomach, proximal small intestine, and colon. Flexible endoscopy has been of particular value in the investigation and treatment of patients with gastrointestinal bleeding, and gastroscopy and/or colonoscopy can find a bleeding source in more than $90 \%$ of these cases. In a small number of patients these examinations do not reveal a cause. Persistent or recurrent bleeding can lead to multiple investigations, hospitalisation, and even surgical interventions, all of which may fail to identify the bleeding source. ${ }^{23}$

Push enteroscopy is valuable in some such patients as it allows examination of a proportion of the proximal jejunum. It can identify bleeding sources from that area and not infrequently may find pathology missed at a previous gastroscopy. ${ }^{4-7}$ Despite substantial improvements to push enteroscopy in the last 10 years $^{8}$ with manufacturers producing longer instruments of $240 \mathrm{~cm}$ with video and overtubes, these instruments can only examine the upper gastrointestinal tract up to the proximal jejunum. Sonde enteroscopes which are approximately $275 \mathrm{~cm}$ in length can reach further into the small intestine. The discomfort experienced by the patient and length of time required for this examination have prevented widespread dissemination of this method, which is rarely performed nowadays. ${ }^{9}$

The small intestine in human adults is over $5 \mathrm{~m}$ in length. A median length of $575 \mathrm{~cm}$ was reported for adults over 20 years old in a pooled series of 1010 necropsies. ${ }^{11}$

Indirect methods to visualise the small bowel in patients with small intestinal bleeding have produced low diagnostic yields of approximately 5-8\% for small bowel follow through or enteroclysis. ${ }^{25}$ Angiography is rarely helpful unless the patient is actively bleeding at a rate of more than 3-5 $\mathrm{ml} / \mathrm{min}^{2512}$ and scintigraphy with technetium labelled red cells is of limited value, particularly in the foregut. ${ }^{13}$ Meckel scans are helpful when positive, but if negative do not exclude a Meckel's diverticulum as a bleeding source. ${ }^{14}$

Improved methods to visualise the entire small intestine might have clinical advantages, especially in this subgroup of patients with undiagnosed gastrointestinal bleeding.

Development of a new radiotelemetry capsule video endoscope, which is small enough to be swallowed $(11 \times 27$ $\mathrm{mm}$ ) and has no external wires, fibreoptic bundles, or cables, ${ }^{15-18}$ made it possible to acquire images of the whole of the small bowel.

The aims of this study were to compare the efficacy and clinical impact of this new wireless capsule endoscopy with push enteroscopy in patients with recurrent bleeding or anaemia when gastroscopy and colonoscopy were negative. The limitations, technical failures, and practical issues involved in using this emerging technology were explored.

\section{MATERIALS, PATIENTS, AND METHODS}

The wireless capsule endoscopes used in this study were the M2A (mouth to anus) capsules with a lifespan of up to 7.5 hours (Given Imaging, Yoqneam, Israel). The device measured $11 \times 27 \mathrm{~mm}$ and weighed $3.7 \mathrm{~g}$. It contained a light source formed from white light emitting diodes, a complementary metal oxide silicon imager containing $256 \times 256$ pixels, a colour television transmitter on an application specific integrated circuit, and two silver oxide batteries all encapsulated in a strong plastic container with a transparent optical dome window. Per patient, approximately 50000 transmitted images from the M2A capsule were received via an array of eight aerials attached to the patient's abdomen and stored on a portable 
Table 1 Volunteer and patients characteristics, and outcome

\begin{tabular}{lll}
\hline & Volunteers & Patients \\
\hline $\mathrm{n}$ & 14 & 50 \\
Age (y) (median (range)) & $45(28-58)$ & $50.3(17-80)$ \\
Sex (M/F) & $8 / 6$ & $21 / 29$ \\
No of investigations & $0.2(0-2)$ & $8(3-17)$ \\
Overt/occult bleeding & & $11 / 39$ \\
Duration of bleeding (y) (median (range)) & & $4.2(0.5-20)$ \\
Transfusion units (units) (median (range)) & & $35.5(0-400)$ \\
No of bleeding causes found with capsule & & $68 \%$ \\
No of bleeding causes found with enteroscopy & $38 \%$ & $38 \%$ \\
No of pathologies found with capsule & & $4 / 3(8 \% / 6 \%)$ \\
No of non-small intestinal sources found with capsule/enteroscopy & & \\
\hline
\end{tabular}

solid state recorder, which is carried on a belt around the patient's waist.

A video SIF Q 140 Olympus (Keymed, Southend, UK) push enteroscope with $240 \mathrm{~cm}$ of working length was used for conventional enteroscopy.

The wireless capsule endoscopy was performed within two weeks prior to push enteroscopy. Wireless capsule and push enteroscopy examinations were performed and reported by different examiners who were unaware of the findings of the other examination. Two endoscopists (AFR and PS) carried out the push enteroscopy examinations. One physician (MM) administered the wireless capsule examinations and read the images. The endoscopist, who had not performed push enteroscopy and was unaware of the clinical details, subsequently reviewed the wireless capsule endoscopy images to reduce bias in interpretation, and to assess agreement in wireless capsule image interpretation.

The study was prospectively planned and ethics committee approval was granted for this study in 1999 and 2000. The human volunteer and clinical trial studies were considered and approved separately.

\section{Volunteers and patients}

Fourteen volunteers aged 21-58 years swallowed the capsule to provide information on image quality, transit, and normal appearance of wireless capsule endoscopy.

Fifty two patients aged 17-80 years ( 29 female, 23 male) with obscure or occult ${ }^{4}$ gastrointestinal bleeding agreed to enter into the clinical study, comparing wireless capsule endoscopy with push enteroscopy. One patient was unable to swallow the capsule. In another the push enteroscope could not be advanced beyond the duodenal bulb so that 50 patients were examined with both techniques (table 1).

Entry criteria demanded that they had been investigated with a negative gastroscopy and colonoscopy prior to the capsule investigation. Relevant concurrent illnesses were present in seven patients: von Willebrand's disease $(n=3)$, and mitral valve replacement due to rheumatic fever, Marfan's syndrome, pulmonary embolism, and dermatomyositis in one patient each. Four patients were on drug treatments which might have contributed to bleeding (two were taking warfarin, one was on long term steroids). Another patient was taking aspirin without realising that this was "a drug".

Most of these patients had been extensively examined with multiple procedures (median $n=8$ ) (table 1) to detect the cause of bleeding prior to this study. The investigations included gastroscopies (median 4, range 2-9), colonoscopies (median 2, range 1-6), barium follow through (median 1 , range 1-3), computed tomography scan (median l, range $0-3$ ), angiography (median 0.4 , range $0-3$ ), Meckel or technetium bleeding scans (median 0.7, range 0-5), laparotomies (median 0.3 , range $0-1$ ), and other (median $0.3,0-1$ ). Forty seven of 50 patients had received transfused blood;15/50 had received more than 100 units of blood. Three patients had received more than 400 units.

All volunteers and patients fasted overnight prior to swallowing the capsules. The capsule was swallowed with water and a few drops of simethicone to reduce bubbling. They were allowed to drink water one hour and to eat 2-3 hours after swallowing the capsule.

Two reviewers independently examined all the wireless capsule images. The third endoscopist arbitrated on any discrepancies in the interpretation of the findings.

\section{Statistical analysis}

For statistical assessment of differences between means and standard deviations, a $\chi^{2}$ test was used. A $p$ value of less than 0.05 was assumed to be statistically significant.

\section{RESULTS}

Fourteen volunteers aged 26-58 years were examined to acquire information on the normal appearance of the small bowel. The appearances of the small bowel found with capsule endoscopy were generally similar to those of push enteroscopy. In six volunteers lymphangiectatic cysts were seen (fig 1). These cysts were also seen in over half of all patients in our series. This appearance is probably a "normal" variation of no clinical significance. In volunteers as well as in patients, progressive darkening of the images was observed as the capsule passed to the distal small bowel. This appearance is probably due to concentration of bile but also to non-absorbed residual particulate food material. In the course of this study due to these dark images, the later seven volunteers were given a colonoscopy type preparation with sodium picolax. Five minute video segments taken at 10, 20, and 30 minutes after passage of the pylorus and at the same time intervals before crossing the ileocaecal valve were scored using a subjective visual analogue scale for brightness, absence of obscuring material, and image quality. The images from the lower small intestine were significantly brighter, contained less obscuring material, and had better image quality in volunteers who had received the preparation than in those who had not $(p<0.05)$. Images in the upper small intestine were not significantly different between the two groups.

Fifty patients with gastroscopy and colonoscopy negative gastrointestinal bleeding and suspected small intestinal source were assessed in this study with both capsule and push enteroscopy. The median number of investigations apart from a gastroscopy and colonoscopy carried out to localise the bleeding in this group of patients prior to this study was 8 (3-17) (table 1).

The wireless capsule examination identified a bleeding source in the small intestine in $34 / 50$ patients $(68 \%)$. These included angiodysplasia ${ }^{16}$ ( fig 2), focal active bleeding ${ }^{8}$ (fig 3), small bowel tumour both located in the ileum ${ }^{2}$ (both carcinoid tumours), apthous ulceration and skip lesions suggestive of 


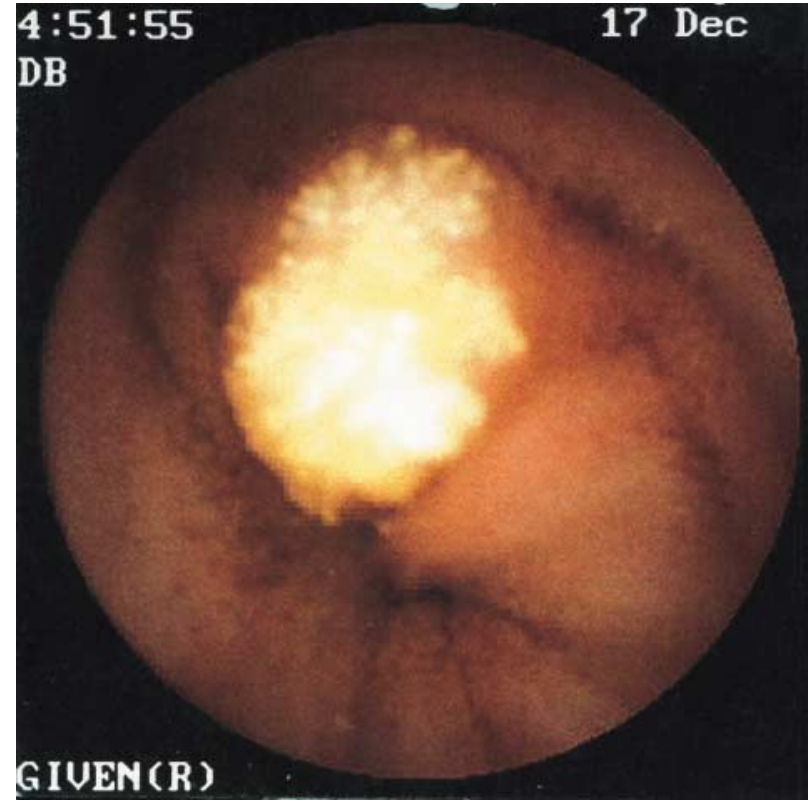

Figure 1 Large lymphangiectatic cyst seen on capsule image in a healthy volunteer

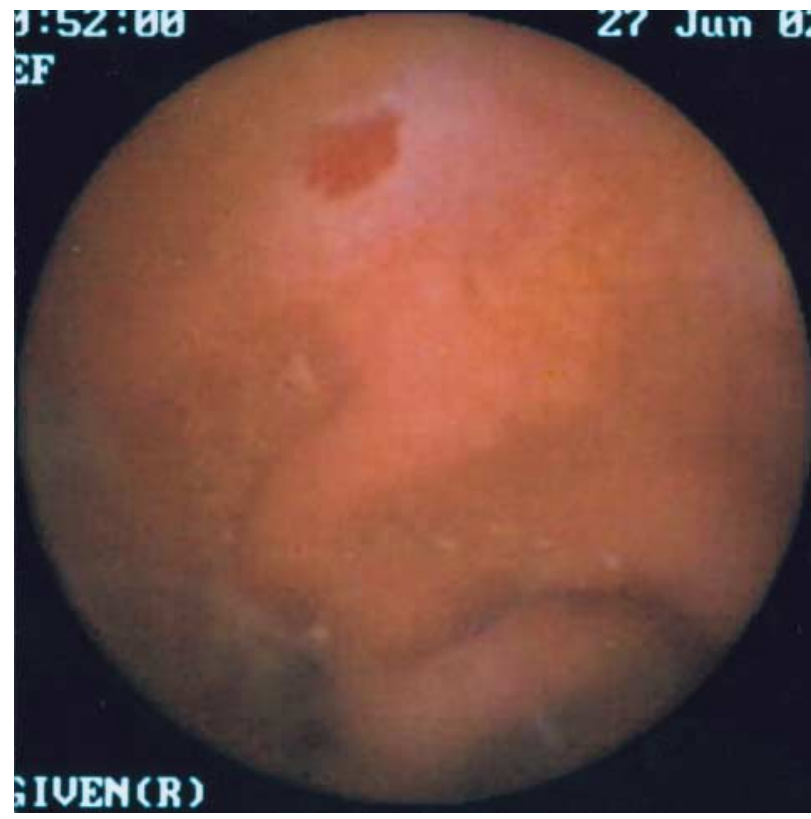

Figure 2 Angiodysplasia in the distal small bowel as a potential bleeding source seen on capsule image in a patient with occult bleeding. The angiodysplasia is located at 12:00 while the lumen of the bowel is hardly seen at 06:00.

Crohn's disease ${ }^{3}$ (fig 4), Meckel's diverticulum, ${ }^{2}$ ulcerating proximal jejunitis, ${ }^{1}$ ileal ulcer, ${ }^{1}$ and ulcer due to intussusception. ${ }^{1}$ The wireless capsule also made some diagnoses outside the small intestine: Cameron erosions in a large hiatus hernia, small gastric angiodysplasia (associated with small intestinal angiodysplasia) in two patients, a gastric ulcer, a caecal polyp, and a caecal angiodysplasia. All gastric abnormalities were confirmed at subsequent push enteroscopy; the colonic abnormalities were confirmed and treated at subsequent colonoscopy. Capsule endoscopy however failed to detect a Cameron lesion in one patient. This was detected at push enteroscopy. The total diagnostic yield of wireless capsule endoscopy was 38/50 (76\%).

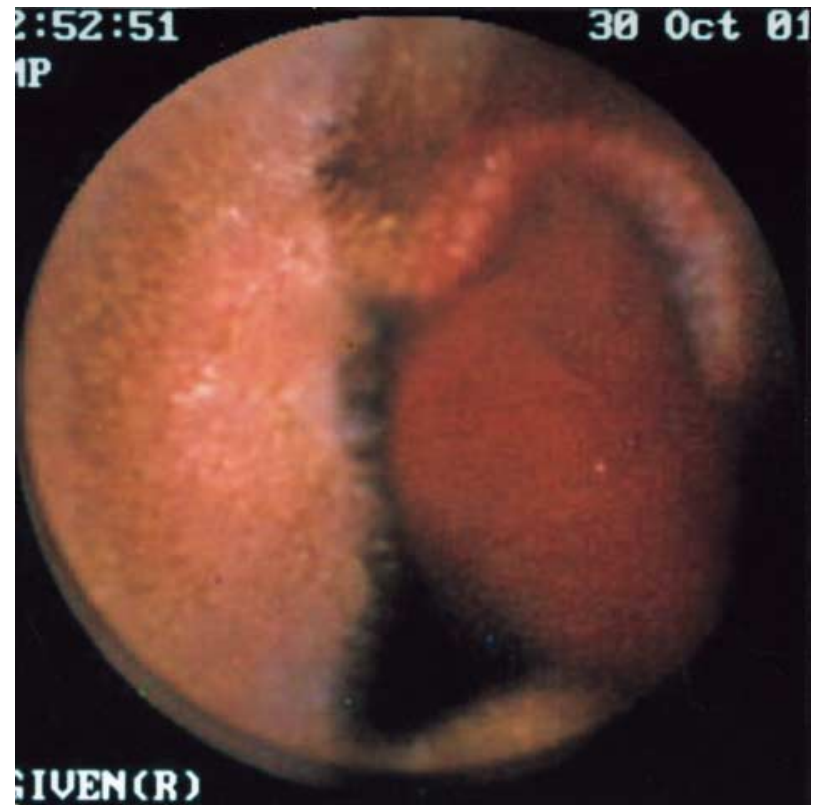

Figure 3 Focal active bleeding in the small bowel seen on capsule image. The dark red bleeding appears in the lumen.

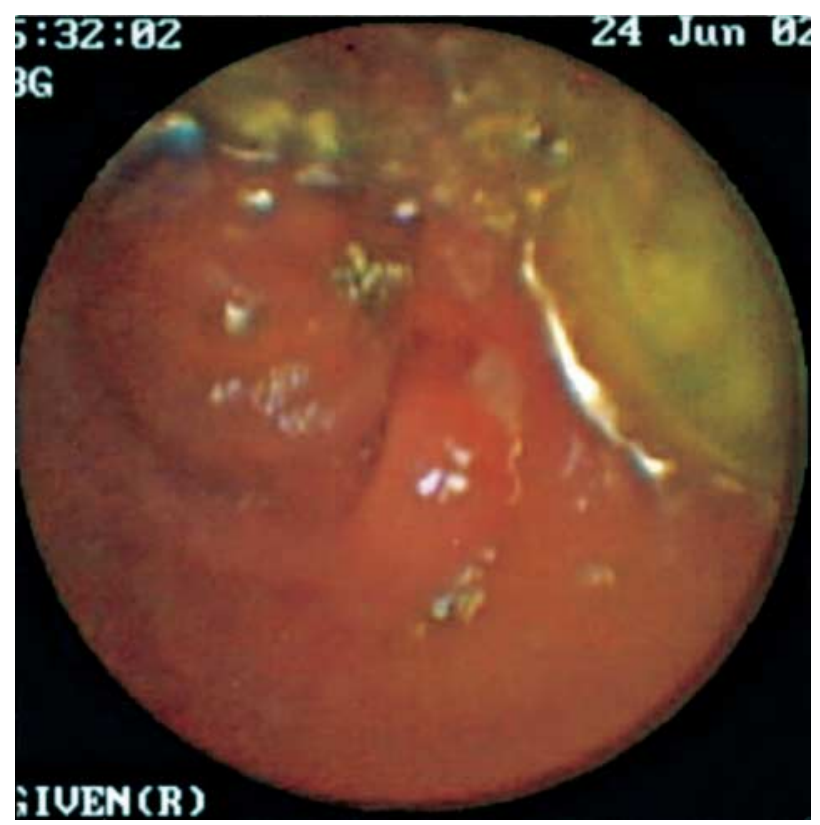

Figure 4 Small apthous lesion seen in the distal small bowel. Several of these lesions were seen in this patient which were suggestive of Crohn's disease.

Push enteroscopy detected angiodysplasia in 10 patients, focal active bleeding in five, and ulcerating jejunitis in one. The diagnostic yield of push enteroscopy to diagnose a bleeding source in the small bowel was 16/50 (32\%). No additional small bowel diagnoses were made by enteroscopy at first attempt. Following a further bleed, on a second enteroscopy an active bleeding from a small angiodysplasia on a fold $10 \mathrm{~cm}$ distal to the papilla was detected in a patient with von Willebrand's disease. As the capsule endoscopy did not detect it and although enteroscopy identified it only on a second occasion, this was considered to be a false negative result of capsule endoscopy. If the additional extraintestinal diagnoses found at push enteroscopy are included, the total diagnostic yield was 19/50 (38\%). The time taken for push enteroscopy was a mean of 22 minutes. An overtube or $x$ ray was not used 
in the course of this examination. Sedation used was a mean dose of $50 \mathrm{mg}$ of pethidine and $6.5 \mathrm{mg}$ of midazolam.

Wireless capsule endoscopy was significantly superior to push enteroscopy in the identification of bleeding sources in the small intestine $\left(34 / 50 v 16 / 50 ; \mathrm{p}<0.05, \chi^{2}\right.$ test $)$. The wireless capsule was also significantly superior to push enteroscopy when the total diagnostic yield was analysed (38/50 v 19/50; $<<0.05$ ).

Wireless capsule endoscopy led to alteration in therapy in $25 / 38$ patients. Seven patients had surgery-six of these had no further bleeding after discharge (Meckel (two), intussusception (one), ileal carcinoid (two), and isolated large jejunal angiodysplasia (one)). One patient with extensive proximal jejunal angiodysplasia had a marked reduction in transfusion requirement. Twelve patients with proximal jejunal angiodysplasia had endoscopic therapy (with bipolar, argon plasma, or heater probe through a push enteroscope). Three patients with probable Crohn's disease were treated with mesalazine and steroids (two subsequent biopsies at colonoscopy showed granulomas and inflammation). Three had other alterations in drug therapy (stopping occult aspirin therapy, reducing warfarin dosage, or starting/increasing proton pump inhibitor treatment).

At a follow up interview two weeks or more after the examination, patients were asked to compare wireless capsule investigation with push enteroscopy and to indicate whether either examination was painful. A total of 49/50 said that they found wireless capsule endoscopy preferable to push enteroscopy; 2/50 found wireless capsule endoscopy uncomfortable but only at the time of swallowing the capsule; and 34/50 found push enteroscopy painful $(\mathrm{p}<0.05)$.

The technical problems encountered were as follows. One capsule remained in the oesophagus for seven hours in a patient with undiagnosed achalasia. This capsule was pushed into the stomach using a flexible endoscope and passed through the patient. Subsequently, another capsule was inserted using a hydraulic delivery device into the duodenum and demonstrated the presence of bleeding jejunal angiodysplasia. Temporary electrical disconnection at the aerial recorder interface was associated with some image loss in three patients. In one patient the battery power ran out at two hours and only a short period of small intestinal imaging was acquired (22 minutes). In seven patients the capsule passed into the pylorus and then returned to the stomach before passing on down the small intestine at least once. In one patient this occurred seven times. The capsule never got stuck in the small intestine or colon. No other serious complications occurred.

The capsule battery expired before reaching the caecum in $16 / 50$ patients $(28 \%)$. In the other patients the capsule delivered images from the right colon for 0-4.5 hours (median 2.20 hours).

The time taken to examine the wireless capsule images was a median of 75 minutes (range 40-120 minutes). With its 22 minutes of examination time, push enteroscopy was significantly quicker in achieving a diagnosis $(\mathrm{p}<0.05)$

In $2 / 38$ patients there was disagreement on interpretation as to the source of bleeding. In both cases this related to the presence or absence of angiodysplasia seen as "red spots" on the images. In one, push enteroscopy confirmed the presence of angiodysplasia. Both reviewers were correct about the site of bleeding in the patients who had subsequent surgery. Both wrongly thought that an appearance, which at surgical pathology was an intussuscepted Meckel's diverticulum, was an intestinal polyp.

\section{DISCUSSION}

Despite careful examination of the upper and lower gastrointestinal tract with flexible endoscopy, the reason and

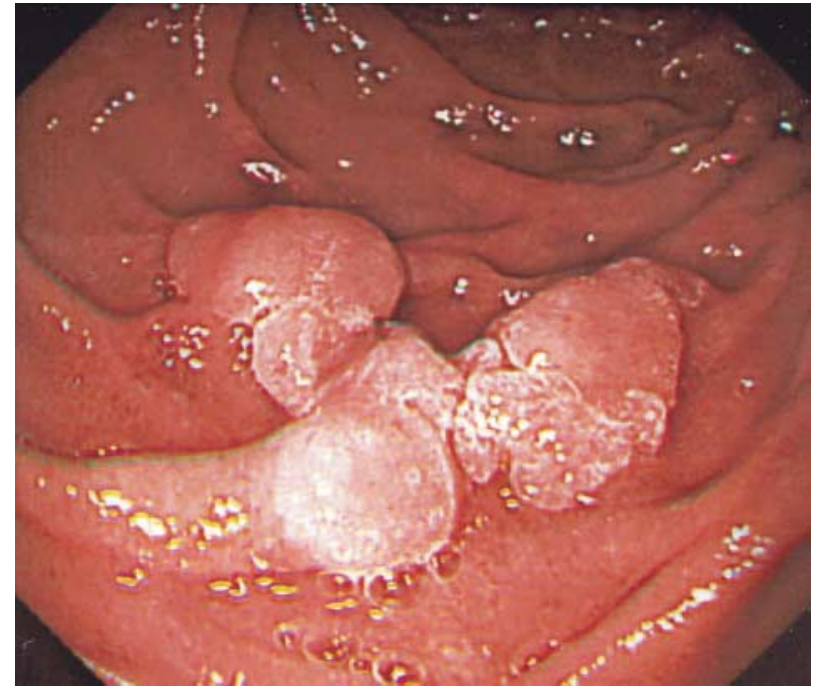

Figure 5 Image captured with push enteroscope GIF Q 140 showing an adenoma in the third part of the duodenum. The image quality of the push enteroscope is superior to that of the wireless capsule (see figs 1-4).

location of occult gastrointestinal bleeding remains unexplained in $30-50 \%$ of patients. ${ }^{2}$ Studies of non-endoscopic imaging techniques in patients with obscure bleeding give generally disappointing results. ${ }^{212-14}$ Development of a wireless endoscopy capsule, which can travel through the whole gut and is moved by the patient's own peristalsis, offers the prospect of painless imaging of the whole of the small intestine.

A study by Lewis and Swain comparing push enteroscopy and capsule endoscopy for occult gastrointestinal bleeding in patients with negative endoscopy and colonoscopy was performed to obtain American Federal Drug Administration approval for the capsule. ${ }^{19}$ In 21 patients with gastroscopy and colonoscopy negative obscure bleeding, capsule endoscopy detected a bleeding site in 11 and enteroscopy in six $(55 \% / 30 \%)$.

In our study with 52 patients, the wireless capsule endoscopy performed similarly well in the investigation of selected patients. It was significantly superior $(68 \% / 32 \%)$ to push enteroscopy in the identification of bleeding abnormalities in the small intestine. In addition to findings in the small bowel, both methods detected pathologies that had been missed at gastroscopy and/or colonoscopy. Several reported series of push enteroscopy $y^{2-8} 20$ show that in patients with recurrent bleeding a source within reach of a gastroscope can be found in $10-25 \%$ of cases. The commonest missed lesions are Cameron erosions in a large hiatus hernia, peptic ulcers, Dieulafoy ulcer, and watermelon stomach, ${ }^{2}$ and the miss rate at colonoscopy both of polyps ${ }^{21}$ and cancers ${ }^{22}$ has also been documented. In this study, the wireless capsule and enteroscopy also made some diagnoses outside the small intestine: Cameron erosions in a large hiatus hernia, small gastric angiodysplasia, a gastric ulcer, a caecal polyp, and a caecal angiodysplasia. All gastric abnormalities were confirmed at push enteroscopy. The miss rate at wireless capsule endoscopy is uncertain at present but is not zero. In one case of recurrent bleeding, an angiodysplasia in the fourth part of the duodenum was missed with the capsule. It was also missed with an initial push enteroscopy but identified and treated on a second approach. It is also true that the number of angiodysplasia identified at push enteroscopy was frequently larger than the number seen during the capsule examination but in patients where the cause of bleeding is uncertain the number of angiodysplasia is less important.

There are some limitations to wireless capsule endoscopy, which is at present a relatively immature technology. The picture quality is not as good as the best quality flexible video 
endoscopy (fig 5). The frame rate with the capsule is lower (2 $v 25$ frames per second), the light intensity cannot yet respond to altering requirements, lesions cannot be washed or repeatedly examined, and the optics are not yet as good.

There is a sense in which comparison with push enteroscopy is unfair. The push enteroscope cannot reach the lower small intestine. The wireless capsule can make diagnoses beyond the reach of push enteroscopy. There may have been a tendency to overestimate the length of the small intestine examined by push enteroscopy when this was the only available endoscopic method. ${ }^{7}$ Patient studies in this series are highly selected, troublesome to manage, expensive, and not very common. Our unit acts as a referral centre for patients with obscure gastrointestinal bleeding and performs 2-3 push enteroscopy examinations each week. Wireless capsule endoscopy is currently the only non-operative method to examine the whole of the small intestine beyond the reach of the push enteroscope as Sonde type enteroscopy has been largely abandoned. ${ }^{9}$

Another limitation of capsule endoscopy at present is the lack of funding as only a few National Health Service Trusts or private insurance companies have as yet agreed to pay for this examination. The disposable capsule currently costs $£ 350$ with a capital cost for the work station, recorder, and aerial belts of $£ 20$ 000. The medical time required to analyse the images is another cost.

It is too early to be dogmatic about algorithms for investigating patients with gastroscopy and colonoscopy negative gastrointestinal bleeding. This study suggests that capsule endoscopy is likely to find more bleeding sources than push enteroscopy. These results are supported by other studies published in abstract form. ${ }^{23-29}$ Push enteroscopy has therapeutic potential unlike the capsule and is currently somewhat more widely available than capsule endoscopy and so perhaps should be considered first at the present time. Repeat gastroscopy and colonoscopy by an experienced operator may well be worth undertaking before investigating the small intestine. Careful history taking, access to all previous imaging information, and good clinical judgement remain important in managing this difficult subgroup of patients with gastrointestinal bleeding.

Although wireless capsule endoscopy has limitations, it is the only imaging method that can provide optical colour images of the lower small intestine painlessly without surgery and has opened a new perspective in the investigation of the causes of bleeding in patients with suspected small intestinal abnormalities. It is as yet an immature technology. Technical improvements need to be encouraged.

\section{CONCLUSIONS}

This study shows that capsule endoscopy can provide small intestinal imaging comparable with push enteroscopy and can diagnose intestinal bleeding sources at sites beyond the reach of push enteroscopes. It was safe, painless, and well tolerated.

\section{Authors' affiliations}

M Mylonaki, A Fritscher-Ravens, P Swain, Department of

Gastroenterology, Royal London Hospital, Whitechapel, London, UK

\section{REFERENCES} 1 Hopkins $\mathbf{H}$, Kapany NS. A flexible fiberscope, using static scanning
Nature $1954 ; 173: 39$.
2 Zuckerman GR, Prakesh C, Askin MP, et al. AGA technical review on the evaluation and management of occult and obscure gastrointestinal bleeding. Gastroenterology 2000;1 18:201-21.

3 Rockey DC. Occult gastrointestinal bleeding. N Engl J Med 1999;341:38-45

4 Hayat M, Axon AT, O'Mahony S. Diagnostic yield and effect on clinical outcomes of push enteroscopy in suspected small-bowel bleeding. Endoscopy 2000;32:369-72

5 Lewis BS. Enteroscopy. Gastrointest Endosc Clin N Am 2000;29:101-117.

6 Lewis BS, Waye J. Chronic gastrointestinal bleeding of obscure origin: role of small bowel enteroscopy. Gastroenterology 1988:94:11 17-20.

7 Davies GR, Gertner DJ, Benson M, et al. Diagnostic, therapeutic and practical advantages of small bowel examination with a push type enteroscope. Gut 1995;37:346-52.

8 Barkin JS, Chong J, Reiner DK. First-generation video enteroscope: fourth-generation push-type small bowel enteroscopy utilizing an overtube. Gastrointest Endosc 1994:40:743-7.

9 Lewis B. Enteroscopy: endangered by the capsule? Endoscopy 2002;34:416-17

10 Seensalu R. The sonde examination. Gastrointest Endosc Clin N Am1999;9:37-59

11 Weaver LT, Austin S, Cole TJ. Small intestinal length: a factor essential for gut adaptation. Gut 1991;32:1321-3.

12 Fiorito JJ, Brandt L, Kozicky O, et al. The diagnostic yield of superior mesenteric angiography: Correlation with the pattern of gastrointestinal bleeding. Am J Gastroenterol 1989;84:878-81.

13 Howarth DM, Tang C, Lees W. The clinical utility if nuclear imaging for the detection of occult gastrointestinal haemorrhage. Nucl Med Commun 2002; 23:591-4

14 Swaniker F, Soldes O, Hirschl RB. The utility of technetium $99 \mathrm{~m}$ pertechnetate scintigraphy in the evaluation of patients with Meckel's diverticulum. J Pediatr Surg 1999:34:760-65

15 Iddan G, Meron G, Glukhovsky A, et al. Wireless capsule endoscopy. Nature 2000;405:417.

16 Gong F, Mills TN, Swain CP. Wireless endoscopy. Gastrointest EndosC 2000;51:725-9.

17 Appleyard M, Fireman F, Glukhovsky A, et al. Randomized comparison of push-enteroscopy and wireless capsule endoscopy in detecting small intestinal lesions in an animal model. Gastroenterology 2000;1 19:1431-8

18 Appleyard M, Glukhovsky A, Swain CP. Wireless capsule diagnostic endoscopy for recurrent small-bowel bleeding. N Engl J Med 2001;34: 232-3

19 Lewis BS, Swain CP. Capsule endoscopy in the evaluation of patients with suspected small intestinal bleeding: Results of a pilot study. Gastrointest Endosc 2002; 56:349-53.

20 Zaman A, Katon RM. Push enteroscopy for obscure gastrointestinal bleeding yields a high incidence of proximal lesions within reach of a standard endoscope. Gastrointest Endosc 1998;47:372-5.

21 Rex DK, Cutler CS, Lemmel GT, et al. Colonoscopic miss rates of adenomas determined by back-to-back colonoscopies. Gastroenterology 1997;112:24-8.

22 Haseman JH, Lemmel GT, Rahmani EY, et al. Failure of colonoscopy to detect colorectal cancer: evaluation of 47 cases in 20 hospitals. Gastrointest Endosc 1997:54:451-5.

23 Delvaux MM, Saurin JC, Gaudin JJ, et al. Comparison of wireless endoscopic capsule and push enteroscopy in patients with obscure gastrointestinal bleeding. Results of a prospective, blinded multicenter trial. Gastrointest Endosc 2002;55:AB88.

24 Remke S, Helou L, Mayer G, et al. Wireless capsule endoscopy and push enteroscopy in chronic gastrointestinal bleeding: A prospective controlled trial. Gastrointest Endosc 2002;55:AB 125

25 Van Gossum A, Erik F, Schmitt A, Deviere J. A prospective comparative study between push-enteroscopy and wireless video capsule in patients with obscure digestive bleeding. Gastrointest Endosc 2002;55:AB 125

26 Jensen DM, Gareth D, Lousuebsakul V, et al. Diagnostic yield of capsule endoscopy in patients with severe $\mathrm{Gl}$ bleeding of obscure origin. Gastrointest Endosc 2002;55:AB 125

27 Pennazio $M$, Santucci R, Rondonotti E, et al. Wireless capsule endoscopy in patients with obscure gastrointestinal bleeding: Results of the Italian multicenter experience. Gastrointest Endosc 2002;55:AB87

28 Lewis B. Capsule endoscopy in clinical practice (abstract). Gastrointest Endosc 2002;55:AB 125

29 Hahne M, Schilling D, Hartmann D, et al. Wireless capsule endoscopy: a new dimension in small bowel diagnostics. Gastrointest Endosc 2002;55:AB 147 